\title{
The Italian Consensus Conference on Pain in Neurorehabilitation: rationale and methodology
}

\author{
Stefano Tamburin' \\ Stefano Paolucci \\ Francesca Magrinelli' \\ Massimo Musicco 2,3 \\ Giorgio Sandrini ${ }^{4,5}$
}

On behalf of the Italian

Consensus Conference on

Pain in Neurorehabilitation (ICCPN)

'Department of Neurological, Biomedical and Movement Sciences, University of Verona, Verona, ${ }^{2}$ Santa Lucia Foundation, IRCCS, Rome, ${ }^{3}$ Institute of Biomedical Technologies (IBT) National Research Council of Italy (CNR), Segrate, Milan, ${ }^{4} \mathrm{C}$. Mondino National Institute of Neurology Foundation, IRCCS, ${ }^{5}$ Department of Brain and Behavioural Sciences, University of Pavia, Pavia, Italy

This article was published in the following Dove Press journal:

Journal of Pain Research

30 May 2016

Number of times this article has been viewed

Abstract: Pain is very common in the neurorehabilitation setting, where it may not only represent a target for treatment but can also negatively influence rehabilitation procedures directly or through the side effects of painkillers. To date, there are neither guidelines nor consensus on how to assess and treat pain in neurorehabilitation. Because of the very scanty pieces of evidence on this topic, the Italian Consensus Conference on Pain in Neurorehabilitation (ICCPN) was promoted under the auspices of different scientific societies. This article illustrates the rationale, methodology, and topics of the ICCPN. The recommendations of the ICCPN will offer some information on how to deal with pain in neurorehabilitation and may represent the starting point for further studies.

Keywords: assessment, consensus conference, neurology, pain, rehabilitation, treatment

\section{Introduction}

A large number of studies document that chronic pain may have an adverse effect on physical, cognitive, and psychosocial functioning. ${ }^{1,2}$ Pain has recently received attention as a secondary complication and has been recognized as a common problem in patients undergoing neurorehabilitation, but its prevalence, severity, and psychosocial impact in this setting are still unknown. ${ }^{3}$

Spinal cord injury, traumatic brain injury, back pain, osteoarthritis, rheumatoid arthritis, multiple sclerosis, stroke, and limb amputation stand among the most common conditions that require inpatient and outpatient rehabilitation care. ${ }^{4}$ Among these diseases, back pain and arthritis, where pain is the leading complaint, were found to cause the highest economic and social burden. ${ }^{5}$ However, the impact of pain in the other common conditions listed above is largely unexplored.

Pain can negatively influence or even impede neurorehabilitation procedures, ${ }^{3}$ and most of the drugs used to treat pain, in particular neuropathic pain, ${ }^{6}$ may have an unfavorable side effect profile and may worsen neurological symptoms and cognition, with largely unknown consequences on recovery processes.

A very recent study explored the characteristics and impact of pain in people with

Correspondence: Stefano Tamburin Department of Neurological, Biomedical and Movement Sciences, University of Verona, Piazzale Scuro 10, I-37/34 Verona, Italy

Tel +3945812 4285/+393475235580

Fax +39458027276

Email stefano.tamburin@univr.it stroke undergoing neurorehabilitation and reported that pain was present in $33 \%$ of the patients. ${ }^{7}$ Moreover, pain influenced rehabilitation treatment and outcomes in $25 \%$ of stroke patients and reduced attention during rehabilitation sessions in $16 \%$ of them, having a negative impact on the quality of life and eventually increasing the costs of the rehabilitation process. ${ }^{7}$ 
A number of guidelines on pain management have been produced and regularly updated in recent years. For example, converging recommendations from different scientific societies help the neurologist or pain clinician to make the diagnosis, assess, ${ }^{8,9}$ and treat neuropathic pain. ${ }^{10-16}$ However, till now, how and to what extent such guidelines apply to the neurorehabilitation setting have not been explored.

The quality of scientific research on the rehabilitative approaches to treat pain is generally considered rather poor, which might be due to several reasons, including the low appeal of studies designed to explore the effect of a given rehabilitation treatment, the rather low impact factor of rehabilitation journals, and the difficulties in designing and conducting a randomized controlled trial (RCT) in rehabilitation. ${ }^{17}$ RCTs are mandatory for scientific evidence but are sometimes not feasible in the rehabilitation field because of the difficulties in designing true sham control conditions, in defining homogeneous patient groups, and in keeping equal duration and intensity of treatments among different centers. ${ }^{17}$

Because of the very scanty pieces of evidence on how to deal with pain in neurorehabilitation, the Italian Consensus Conference on Pain in Neurorehabilitation (ICCPN) was promoted by the Italian Society of Neurorehabilitation (Società Italiana di Riabilitazione Neurologica) and the Italian Society of Physical and Rehabilitative Medicine (Società Italiana di Medicina Fisica e Riabilitativa). This article illustrates the rationale, the methodology, and the topics of the ICCPN.

\section{Methods}

The methodology of the ICCPN was based on the Italian guidelines for organizing a consensus conference, ${ }^{18}$ the Italian guidelines on stroke (Stroke Prevention and Educational Awareness Diffusion), ${ }^{19}$ and the Consensus Conference on neuropsychological rehabilitation in adult patients. ${ }^{17}$

Most of the current guidelines and evidence on the pharmacological and nonpharmacological treatment of pain may be difficult to transfer to the field of neurorehabilitation, and sound evidence is lacking for the majority of rehabilitative treatments of pain. For these reasons, we considered evidence derived from experimental, observational, case-control, and other types of studies, as well as the opinion of experts.

\section{ICCPN task force}

The ICCPN task force was composed of a promoter committee, a technical-scientific committee, and a jury. The task force of the ICCPN was formed by experts from
Società Italiana di Riabilitazione Neurologica, Società Italiana di Medicina Fisica e Riabilitativa, and from other Italian scientific societies interested in neurology, rehabilitation, and pain, including (in alphabetical order) the Italian Association for the Study of Pain (Associazione Italiana per lo Studio del Dolore), the Italian Association of Physiotherapists (Associazione Italiana Fisioterapisti), the Italian Society for the Study of Headache (Società Italiana per lo Studio delle Cefalee), the Italian Society of Clinical Neurophysiology (Società Italiana di Neurofisiologia Clinica), the Italian Society of Neurology (Società Italiana di Neurologia) and their study groups on neurosciences and pain (Neuroscienze e Dolore) and movement disorders (Accademia LIMPE - DISMOV), the Italian Society of Neuropsychiatry of the Infancy and the Adolescence (Società Italiana di Neuropsichiatria dell'Infanzia e dell'Adolescenza), the Italian Society of Pain Clinicians (Società Italiana dei Clinici del Dolore, FederDolore), the Italian Society of Palliative Care (Società Italiana di Cure Palliative), and the Italian Society of Rheumatology (Società Italiana di Reumatologia).

\section{ICCPN topics and working groups}

The topics of the ICCPN were divided into 27 working groups, which were incorporated into seven main paragraphs, three of which dealt with general issues and the remaining four dealt with specific clinical conditions that may be encountered in the neurorehabilitation setting. Common diseases with nociceptive pain were also included, because they may coexist with neurological conditions, especially in the elderly. The main paragraphs and working groups are listed as follows:

1. Diagnosing and assessing pain in neurorehabilitation (from translational research to the clinical setting): 1. translation research on pain; 2. neuropathic, nociceptive, and mixed pain; and 3. instrumental evaluation of pain.

2. The role of sex and psychosocial factors in pain in neurorehabilitation: 4 . sex-related pain biomarkers; 5 . the psychiatric comorbidity and the anthropological and cultural dimensions of pain; and 6. the psychological dimension of pain.

3. Pharmacological and nonpharmacological strategies in the integrated approach to pain in neurorehabilitation: 7. pharmacological, interventional, physical, and complementary therapies for the treatment of pain; 8. coping strategies, psychotherapy, and cognitive-behavioral therapies for pain; and 9. the role of the physical therapist. 
4. Assessing and treating pain associated with stroke, multiple sclerosis, cerebral palsy, spinal lesions, and spasticity: 10. pain and spasticity; 11. pain in acute and chronic stroke; 12. pain in multiple sclerosis; 13. pain in spinal cord injury; and 14. pain in cerebral palsy and in pediatric patients.

5. Pain in degenerative, posttraumatic, infectious, and neoplastic central nervous system diseases: 15 . pain in movement disorders; 16. pain in motor neuron disease; 17. pain in chronic disorders of consciousness and dementia; 18. pain in oncology and neurooncology; and 19. pain in neuroinfectious disease.

6. Pain in neuromuscular disorders and neuropathies: 20. pain in plexopathy, radiculopathy, and mononeuropathy; 21. deafferentation and phantom limb pain; and 22. pain in peripheral neuropathies.

7. Headache, low back pain, and other nociceptive and mixed pain conditions: 23. headache and facial pain; 24. low back pain and failed back surgery syndrome; 25. osteoarticular pain; 26. myofascial pain and fibromyalgia; and 27. chronic pelvic pain.

Each working group had a chairperson, and their members defined a number of questions to be answered by the ICCPN. Conflicts of interests were declared at the time of the formation of the groups, and the chairperson was chosen among those declaring no conflict of interests.

\section{Bibliographic search and collection of papers}

For the bibliographic search, the following search engines were used: Pubmed ${ }^{20}$ Medline, and Embase ${ }^{21}$ Sets of specific and sensitive keywords or a combination of keywords were chosen, and the keywords of each group were checked centrally by a steering committee before launching the search. When possible, MeSH terms were used. The search was extended to a time period of 30 years and 20 years, respectively, for pharmacological and nonpharmacological studies. An example of the search strategy used by one of the working groups is reported in Table 1.

All the pertinent meta-analyses, guidelines, and reviews were considered, including the Cochrane Library ${ }^{23}$ Meta-analyses were always collected and included. Previous guidelines and recommendations were sought from search engines and other sources, including national and international scientific organizations, patient organizations, and national or supranational health-related bodies. The conclusion of the ICCPN task force relied on quality-assured scientific data, and previ- ous guidelines and recommendations were evaluated using the Appraisal of Guidelines for Research and Evaluation checklist $^{24}$ and eventually adopted in part or partially. ${ }^{25}$ The conclusions of the reviews were critically evaluated on the basis of the scientific quality of the original papers, ${ }^{25}$ which could serve as a source of additional data. Unpublished RCTs were searched on clinical trial registries ${ }^{26,27}$ or on pharmacological industries' websites. Data from nonrefereed journals, books, or other publications were considered only upon the judgment of the task force members.

\section{Data evaluation and scoring of evidence}

After bibliographic search and collection of papers, the full papers, including those in press, were read and data were collected from the papers themselves. ${ }^{25}$ The evidence pertaining to each workgroup topic was evaluated and scored according to the Oxford 2011 Levels of Evidence. ${ }^{28}$ These levels stipulate a gradient from the best (level 1) to the worst (level 5) evidence for systematic reviews, RCTs, cohort studies, case series, and mechanistic reasoning. ${ }^{28}$ The bibliographic information, conclusions, and level of evidence of all the papers were tabulated in worksheets.

\section{Proposal of the recommendations and evaluation by the jury}

Each group prepared a final report with the defined questions and their answers, which were presented as recommendations with different grading strengths. The reports contained also summary tables and worksheets with tabulated data from original papers with levels of evidence. Each report included a structured summary with the main conclusions. Existing guidelines and/or consensus conferences prepared by other organizations, where appropriate, were adopted in part or whole with acknowledgment and respect for copyright. The format of each report was as follows: title, authors, structured abstract, objectives, background, search strategy, method for reaching consensus, results, recommendations, conflicts of interest, and references. The reports of all the groups were evaluated by the jury, and, when necessary, discussed with each group before preparing the final recommendations and strength grading.

The levels of the strength of recommendations were evaluated according to A, B, C, D, and good practice point score. ${ }^{17,19}$ The top level was A and referred to high-quality RCTs, and the worst level was good practice point and referred to the best approach based on expert opinion, in the absence of any evidence from literature. ${ }^{17}$ 
Table I The search strategy used by working group 8 (coping strategies, psychotherapy, and cognitive-behavioral therapies for pain) for three main search engines used for the bibliographic search ${ }^{22}$

\begin{tabular}{|c|c|c|}
\hline Pubmed & Embase & $\begin{array}{l}\text { Cochrane Database of Systematic } \\
\text { Reviews }\end{array}$ \\
\hline 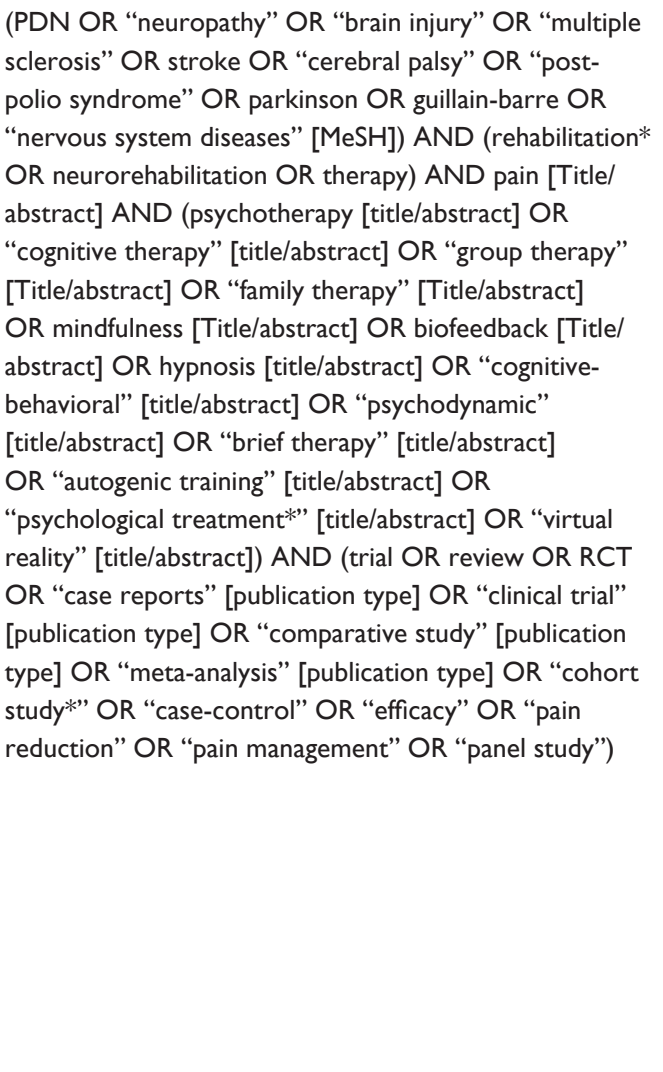 & $\begin{array}{l}\text { Neurologic disease'/exp OR “neurologic disease” } \\
\text { AND pain:ab,ti AND (psychotherapy:ab,ti } \\
\text { OR “cognitive therapy”:ab,ti OR “behavioral } \\
\text { therapy”:ab,ti OR “cognitive-behavioral”:ab,ti } \\
\text { OR mindfulness:ab,ti OR hypnosis:ab,ti OR “brief } \\
\text { therapy”:ab,ti OR “psychodynamic therapy”:ab,ti } \\
\text { OR “acceptance therapy”:ab,ti OR “autogenic } \\
\text { training”:ab,ti OR biofeedback:ab,ti OR “virtual } \\
\text { reality”:ab,ti OR “psychological treatment”:ab,ti) }\end{array}$ & $\begin{array}{l}\text { 2: Pain and (psychotherapy OR } \\
\text { "cognitive therapy" OR "behavioral } \\
\text { therapy" OR "cognitive-behavioral } \\
\text { therapy" OR "hypnosis" or biofeedback } \\
\text { or "psychodynamic" OR "brief } \\
\text { therapy" OR "acceptance therapy" OR } \\
\text { "family therapy" OR "virtual reality") } \\
\text { 3: I and } 2\end{array}$ \\
\hline
\end{tabular}

\section{Writing and diffusion of ICCPN recommendations}

The conclusions of the ICCPN will be written in English for publication in a peer-reviewed journal (expected time of publication: second half of 2016) and translated in Italian for a wider diffusion. In addition to the print copies, the conclusions will be made freely downloadable on a specific website and presented to national and international congresses. An audit on the ICCPN recommendations, including stakeholders (association of patients, health technology producers, health administrators), will follow their publication and diffusion.

\section{Conclusion}

The conclusions and recommendations of the ICCPN will represent a first step for answering the still open question of how to deal with pain in the setting of neurorehabilitation. Apart from offering some practical information on the evaluation and treatment of pain in this specific setting, they may represent the starting point for further studies, which hopefully will reach a high enough quality level to move from consensus conference conclusions to true guidelines.

\section{Acknowledgments}

Support for this study was kindly provided through unrestricted grants by Allergan and Grunenthal. The following authors, who are listed in alphabetical order and collaborators contributed to the work of the ICCPN: Michela Agostini (Neurorehabilitation Department, Foundation IRCCS San Camillo Hospital, Venice, Italy), Enrico Alfonsi (C. Mondino National Institute of Neurology Foundation, IRCCS, Pavia, Italy), Anna Maria Aloisi (Department of Medicine, Surgery and Neuroscience, University of Siena, Siena, Italy), Elena Alvisi (Department of Brain and Behavioural Sciences, University of Pavia, Pavia, Italy), Irene Aprile (Don Gnocchi Foundation, Milan, Italy), Michela Armando (Department of Neuroscience and Neurorehabilitation, Bambin Gesù Children's Hospital, IRCCS, Rome, Italy), Micol Avenali (C. Mondino National Institute of Neurology Foundation, IRCCS, Pavia, Italy; Department of Brain and Behavioural 
Sciences, University of Pavia, Pavia, Italy), Eva Azicnuda (Santa Lucia Foundation, IRCCS, Rome, Italy), Francesco Barale (Department of Brain and Behavioural Sciences, University of Pavia, Pavia, Italy), Michelangelo Bartolo (Neurorehabilitation Unit, IRCCS INM Neuromed, Pozzilli, Italy), Roberto Bergamaschi (C. Mondino National Institute of Neurology Foundation, IRCCS, Pavia, Italy), Mariangela Berlangieri (Department of Brain and Behavioural Sciences, University of Pavia, Pavia, Italy), Vanna Berlincioni (Department of Brain and Behavioural Sciences, University of Pavia, Pavia, Italy), Laura Berliocchi (Department of Health Sciences, University "Magna Graecia” of Catanzaro, Catanzaro, Italy), Eliana Berra (C. Mondino National Institute of Neurology Foundation, IRCCS, Pavia, Italy), Giulia Berto (Department of Neurological and Movement Sciences, University of Verona, Verona, Italy), Silvia Bonadiman (Department of Neurological and Movement Sciences, University of Verona, Verona, Italy), Sara Bonazza (Department of Surgery, University of Verona, Verona, Italy), Federica Bressi (Campus Bio-Medico University, Rome, Italy), Annalisa Brugnera (Department of Neurological and Movement Sciences, University of Verona, Verona, Italy), Stefano Brunelli (Santa Lucia Foundation, IRCCS, Rome, Italy), Maria Gabriella Buzzi (Santa Lucia Foundation, IRCCS, Rome, Italy), Carlo Cacciatori (Department of Neurological and Movement Sciences, University of Verona, Verona, Italy), Andrea Calvo (Rita Levi Montalcini Department of Neuroscience, University of Turin, Turin, Italy), Cristina Cantarella (Physical and Rehabilitation Medicine Unit, Tor Vergata University, Rome, Italy), Augusto Caraceni (Palliative Care, Pain Therapy and Rehabilitation, Fondazione IRCCS Istituto Nazionale dei Tumori di Milano, Milan, Italy), Roberto Carone (NeuroUrology Department, City Hospital Health and Science of the City of Turin, Turin, Italy), Elena Carraro (Neuropediatric Rehabilitation Unit, E. Medea Scientific Institute, Conegliano, Italy), Roberto Casale (Department of Clinical Neurophysiology and Pain Rehabilitation Unit, Foundation Salvatore Maugeri IRCCS, Montescano, Italy), Paola Castellazzi (Department of Neurological and Movement Sciences, University of Verona, Verona, Italy), Gianluca Castelnuovo (Psychology Research Laboratory, Istituto Auxologico Italiano IRCCS, Ospedale San Giuseppe, Verbania, Italy; Department of Psychology, Catholic University of Milan, Milan, Italy), Adele Castino (ASL of the Province of Lodi, Lodi, Italy), Rosanna Cerbo (Hub Terapia del Dolore Regione Lazio, Policlinico Umberto I, Sapienza University, Rome, Italy), Adriano Chiò (Rita Levi Montalcini Department of Neuroscience, University of Turin, Turin, Italy), Cristina
Ciotti (Physical and Rehabilitation Medicine Unit, Tor Vergata University, Rome, Italy), Carlo Cisari (Department of Health Sciences, Università del Piemonte Orientale, Novara, Italy), Daniele Coraci (Department of Orthopaedic Science, Sapienza University, Rome, Italy), Elena Dalla Toffola (Department of Clinical, Surgical, Diagnostic and Pediatric Sciences, University of Pavia, Pavia, Italy; IRCCS Policlinico San Matteo Foundation, Pavia; Italy), Giovanni Defazio (Department of Basic Medical Sciences, Neuroscience and Sensory Organs, Aldo Moro University of Bari, Bari, Italy), Roberto De Icco (C. Mondino National Institute of Neurology Foundation, IRCCS, Pavia, Italy; Department of Brain and Behavioural Sciences, University of Pavia, Pavia, Italy), Ubaldo Del Carro (Section of Clinical Neurophysiology and Neurorehabilitation, San Raffaele Hospital, Milan, Italy), Andrea Dell'Isola (Department of Health Sciences, Università del Piemonte Orientale, Novara, Italy), Antonio De Tanti (Cardinal Ferrari Rehabilitation Center, Santo Stefano Rehabilitation Institute, Fontanellato, Italy), Mariagrazia D'Ippolito (Santa Lucia Foundation, IRCCS, Rome, Italy), Elisa Fazzi (Childhood and Adolescence Neurology and Psychiatry Unit, City Hospital, Brescia, Italy; Department of Clinical and Experimental Sciences, University of Brescia, Brescia, Italy), Adriano Ferrari (Children Rehabilitation Unit, IRCCS Arcispedale S. Maria Nuova, Reggio Emilia, Italy), Sergio Ferrari (Department of Neurological and Movement Sciences, University of Verona, Verona, Italy), Francesco Ferraro (Section of Neuromotor Rehabilitation, Department of Neuroscience, Azienda Ospedaliera Carlo Poma, Mantova, Italy), Fabio Formaglio (Pain Medicine Center, Casa di Cura San Pio X, Opera San Camillo Foundation, Milan, Italy), Rita Formisano (Santa Lucia Foundation, IRCCS, Rome, Italy), Simone Franzoni (Poliambulanza Foundation Istituto Ospedaliero, Geriatric Research Group, Brescia, Italy), Francesca Gajofatto (Department of Neurological and Movement Sciences, University of Verona, Verona, Italy), Marialuisa Gandolfi (Department of Neurological and Movement Sciences, University of Verona, Verona, Italy), Barbara Gardella (IRCCS Policlinico San Matteo Foundation, Pavia; Italy), Pierangelo Geppetti (Department of Health Sciences, Section of Clinical Pharmacology and Oncology, University of Florence, Florence, Italy), Alessandro Giammò (NeuroUrology Department, City Hospital Health and Science of the City of Turin, Turin, Italy), Raffaele Gimigliano (Department of Physical and Mental Health, Second University of Naples, Naples, Italy), Emanuele Maria Giusti (Department of Psychology, Catholic University of Milan, Milan, Italy), 
Elena Greco (Department of Neurological and Movement Sciences, University of Verona, Verona, Italy), Valentina Ieraci (Department of Oncology and Neuroscience, University of Turin, City Hospital Health and Science of the City of Turin, Turin, Italy), Marco Invernizzi (Department of Health Sciences, Università del Piemonte Orientale, Novara, Italy), Marco Jacopetti (University of Parma, Parma, Italy), Marco Lacerenza (Pain Medicine Center, Casa di Cura San Pio X, Opera San Camillo Foundation, Milan, Italy), Silvia La Cesa (Department of Neurology and Psychiatry, University of Rome Sapienza, Rome, Italy), Davide Lobba (Department of Neurological and Movement Sciences, University of Verona, Verona, Italy), Gian Mauro Manzoni (Psychology Research Laboratory, Istituto Auxologico Italiano IRCCS, Ospedale San Giuseppe, Verbania, Italy; Department of Psychology, Catholic University of Milan, Milan, Italy), Francesca Magrinelli (Department of Neurological and Movement Sciences, University of Verona, Verona, Italy), Silvia Mandrini (Department of Clinical, Surgical, Diagnostic and Pediatric Sciences, University of Pavia, Pavia, Italy), Umberto Manera (Rita Levi Montalcini Department of Neuroscience, University of Turin, Turin, Italy), Paolo Marchettini (Pain Medicine Center, Hospital San Raffaele, Milan, Italy), Enrico Marchioni (C. Mondino National Institute of Neurology Foundation, IRCCS, Pavia, Italy), Sara Mariotto (Department of Neurological and Movement Sciences, University of Verona, Verona, Italy), Andrea Martinuzzi (Neuropediatric Rehabilitation Unit, E. Medea Scientific Institute, Conegliano, Italy), Marella Masciullo (Santa Lucia Foundation, IRCCS, Rome, Italy), Susanna Mezzarobba (Department of Medicine, Surgery and Health Sciences, University of Trieste, Trieste, Italy), Danilo Miotti (Palliative Care and Pain Therapy Unit, Fondazione Salvatore Maugeri IRCCS, Scientific Institute of Pavia, Pavia, Italy), Angela Modenese (Department of Neurological and Movement Sciences, University of Verona, Verona, Italy), Marco Molinari (Santa Lucia Foundation, IRCCS, Rome, Italy), Salvatore Monaco (Department of Neurological and Movement Sciences, University of Verona, Verona, Italy), Giovanni Morone (Santa Lucia Foundation, IRCCS, Rome, Italy), Rossella Nappi (Department of Clinical, Surgical, Diagnostic and Pediatric Sciences, University of Pavia, Pavia, Italy; IRCCS Policlinico San Matteo Foundation, Pavia; Italy), Stefano Negrini (Don Gnocchi Foundation, Milan, Italy; Department of Clinical and Experimental Sciences, University of Brescia, Brescia, Italy), Andrea Pace (Neuro-Oncology Unit, Regina Elena National Cancer Institute, Rome, Italy), Luca Padua (Don Gnocchi Foundation, Milan, Italy; Institute of Neurology, Catholic University, Rome, Italy), Emanuela Pagliano (Developmental Neurology Unit, C. Besta Neurological Institute Foundation, Milan, Italy), Valerio Palmerini (Hub Terapia del Dolore Regione Lazio, Policlinico Umberto I, Sapienza University, Rome, Italy), Stefano Paolucci (Santa Lucia Foundation, IRCCS, Rome, Italy), Costanza Pazzaglia (Don Gnocchi Foundation, Milan, Italy), Cristiano Pecchioli (Don Gnocchi Foundation, Milan, Italy), Alessandro Picelli (Department of Neurological and Movement Sciences, University of Verona, Verona, Italy), Carlo Adolfo Porro (Department of Biomedical, Metabolic and Neural Sciences, University of Modena and Reggio Emilia, Modena, Italy), Daniele Porru (IRCCS Policlinico San Matteo Foundation, Pavia; Italy), Marcello Romano (Neurology Unit, Azienda Ospedaliera Ospedali Riuniti Villa Sofia Cervello, Palermo, Italy), Laura Roncari (Department of Neurological and Movement Sciences, University of Verona, Verona, Italy), Riccardo Rosa (Hub Terapia del Dolore Regione Lazio, Policlinico Umberto I, Sapienza University, Rome, Italy), Marsilio Saccavini (ASL 2 Bassa Friulana-Isontina, Italy), Paola Sacerdote (Department of Pharmacological and Biomolecular Sciences, University of Milano, Milano, Italy), Giorgio Sandrini (C. Mondino National Institute of Neurology Foundation, IRCCS, Pavia, Italy; Department of Brain and Behavioural Sciences, University of Pavia, Pavia, Italy), Donatella Saviola (Cardinal Ferrari Rehabilitation Center, Santo Stefano Rehabilitation Institute, Fontanellato, Italy), Angelo Schenone (Department of Neuroscience, Rehabilitation, Ophthalmology, Genetics, Maternal and Child Health [DiNOGMI], University of Genoa, Genoa, Italy), Vittorio Schweiger (Department of Surgery, University of Verona, Verona, Italy), Giorgio Scivoletto (Santa Lucia Foundation, IRCCS, Rome, Italy), Nicola Smania (Department of Neurological and Movement Sciences, University of Verona, Verona, Italy), Claudio Solaro (Neurology Unit, ASL3, Genova, Italy), Vincenza Spallone (Department of Systems Medicine, University of Rome Tor Vergata, Rome, Italy), Isabella Springhetti (Functional Recovery and Rehabilitation Unit, IRCCS Fondazione S. Maugeri, Pavia, Italy), Stefano Tamburin (Department of Neurological and Movement Sciences, University of Verona, Verona, Italy), Cristina Tassorelli (C. Mondino National Institute of Neurology Foundation, IRCCS, Pavia, Italy; Department of Brain and Behavioural Sciences, University of Pavia, Pavia, Italy), Michele Tinazzi (Department of Neurological and Movement Sciences, University of Verona, Verona, Italy), Rossella Togni (Department of Clinical, Surgical, Diagnostic and Pediatric Sciences, University of Pavia, 
Pavia, Italy), Monica Torre (Santa Lucia Foundation, IRCCS, Rome, Italy), Riccardo Torta (Department of Oncology and Neuroscience, University of Turin, City Hospital Health and Science of the City of Turin, Turin, Italy), Marco Traballesi (Santa Lucia Foundation, IRCCS, Rome, Italy), Marco Tramontano (Santa Lucia Foundation, IRCCS, Rome, Italy), Andrea Truini (Department of Neurology and Psychiatry, University of Rome Sapienza, Rome, Italy), Valeria Tugnoli (Neurological Unit, University Hospital of Ferrara, Ferrara, Italy), Andrea Turolla (Neurorehabilitation Department, Foundation IRCCS San Camillo Hospital, Venice, Italy), Gabriella Vallies (Department of Neurological and Movement Sciences, University of Verona, Verona, Italy), Elisabetta Verzini (Department of Neurological and Movement Sciences, University of Verona, Verona, Italy), Mario Vottero (Neuro-Urology Department, City Hospital Health and Science of the City of Turin, Turin, Italy), Paolo Zerbinati (Neuro-orthopaedic Program, Hand Surgery Department, Santa Maria Hospital MultiMedica, Castellanza, Italy).

\section{Disclosure}

The authors report no conflicts of interest in this work.

\section{References}

1. Breivik H, Collett B, Ventafridda V, Cohen R, Gallacher D. Survey of chronic pain in Europe: prevalence, impact on daily life, and treatment. Eur J Pain. 2006;10(4):287-333.

2. Tamburin S, Maier A, Schiff S, et al. Cognition and emotional decisionmaking in chronic low back pain: an ERPs study during Iowa gambling task. Front Psychol. 2014;5:1350.

3. Benrud-Larson LM, Wegener ST. Chronic pain in neurorehabilitation populations: prevalence, severity and impact. NeuroRehabilitation. 2000;14(3):127-137.

4. Chan L, Koepsell TD, Deyo RA, et al. The effect of Medicare's payment system for rehabilitation hospitals on length of stay, charges, and total payments. N Engl J Med. 1997;337(14):978-985.

5. Ma VY, Chan L, Carruthers KJ. Incidence, prevalence, costs, and impact on disability of common conditions requiring rehabilitation in the United States: stroke, spinal cord injury, traumatic brain injury, multiple sclerosis, osteoarthritis, rheumatoid arthritis, limb loss, and back pain. Arch Phys Med Rehabil. 2014;95(5):986-995.e1.

6. Magrinelli F, Zanette G, Tamburin S. Neuropathic pain: diagnosis and treatment. Pract Neurol. 2013;13(5):292-307.

7. Aprile IG, Briani C, Pazzaglia C, Cecchi F, Negrini S, Padua L. Pain in stroke patients: characteristics and impact on the rehabilitation treatment. A multicenter cross-sectional study. Eur J Phys Rehabil Med. 2015;51(6):725-736.

8. Cruccu G, Sommer C, Anand P, et al. EFNS guidelines on neuropathic pain assessment: revised 2009. Eur J Neurol. 2010;17(8):1010-1018.

9. Haanpää M, Attal N, Backonja M, et al. NeuPSIG guidelines on neuropathic pain assessment. Pain. 2011;152(1):14-27.

10. Cruccu G, Aziz TZ, Garcia-Larrea L, et al. EFNS guidelines on neurostimulation therapy for neuropathic pain. Eur J Neurol. 2007; 14(9):952-970.
11. Dworkin RH, O'Connor AB, Backonja M, et al. Pharmacologic management of neuropathic pain: evidence-based recommendations. Pain. 2007;132(3):237-251.

12. Attal N, Cruccu G, Baron R, et al; European Federation of Neurological Societies. EFNS guidelines on the pharmacological treatment of neuropathic pain: 2010 revision. Eur J Neurol. 2010;17(9):1113-e88.

13. Finnerup NB, Sindrup SH, Jensen TS. The evidence for pharmacological treatment of neuropathic pain. Pain. 2010;150(3):573-581.

14. Dworkin RH, O'Connor AB, Kent J, et al; International Association for the Study of Pain Neuropathic Pain Special Interest Group. Interventional management of neuropathic pain: NeuPSIG recommendations. Pain. 2013;154(11):2249-2261.

15. Moulin D, Boulanger A, Clark AJ, et al; Canadian Pain Society. Pharmacological management of chronic neuropathic pain: revised consensus statement from the Canadian Pain Society. Pain Res Manag. 2014;19(6):328-335.

16. Finnerup NB, Attal N, Haroutounian S, et al. Pharmacotherapy for neuropathic pain in adults: a systematic review and meta-analysis. Lancet Neurol. 2015;14(2):162-173.

17. Làdavass E, Paolucci S, Umiltà C. Reasons for holding a consensus conference on neuropsychological rehabilitation in adult patients. Eur J Phys Rehabil Med. 2011;47(1):91-99.

18. Candiani G, Colombo C, Daghini R, et al [webpage on the Internet]. Manuale metodologico. Come organizzare una conferenza di consenso, 2009-2013. Available from: http://www.snlg-iss.it/cms/files/manuale_ metodologico_consensus_0.pdf. Accessed August 10, 2015.

19. spread.it [homepage on the Internet]. Stroke Prevention and Educational Awareness Diffusion - SPREAD. Ictus cerebrale: linee guida italiane di prevenzione e trattamento. Available from: www.spread.it. Accessed August 10, 2015.

20. Pubmed. Bethesda, MD: US National Library of Medicine National Institutes of Health [homepage on the Internet]. Available from http:// www.ncbi.nlm.nih.gov/pubmed. Accessed August 10, 2015.

21. Embase. Amsterdam, NL: Elsevier B.V. [homepage on the Internet]. Available from http://store.elsevier.com/embase. Accessed August 10, 2015.

22. Castelnuovo G, Giusti EM, Manzoni GM, et al. Psychological Treatments and Psychotherapies in the Neurorehabilitation of Pain: Evidences and Recommendations from the Italian Consensus Conference on Pain in Neurorehabilitation. Front Psychol. 2016;7:115. doi: 10.3389/ fpsyg.2016.00115.

23. The Cochrane Library. John Wiley \& Sons, Inc. [homepage on the Internet]. Available from http://www.cochranelibrary.com. Accessed August 10, 2015.

24. Brouwers MC, Kho ME, Browman GP, et al; AGREE Next Steps Consortium. AGREE II: advancing guideline development, reporting and evaluation in health care. CMAJ. 2010;182(18):E839-E842.

25. Brainin M, Barnes M, Baron JC, et al; Guideline Standards Subcommittee of the EFNS Scientific Committee. Guidance for the preparation of neurological management guidelines by EFNS scientific task forces revised recommendations 2004. Eur J Neurol. 2004;11(9):577-581.

26. ClinicalTrials.gov. Bethesda, MD: US National Library of Medicine National Institutes of Health [homepage on the Internet]. Available from https://clinicaltrials.gov/ct2/home. Accessed August 10, 2015.

27. EU Clinical Trials Register. London, UK: European Medicines Agency homepage on the Internet]. Available from https://www. clinicaltrialsregister.eu. Accessed August 10, 2015.

28. Hawick J, Chalmers I, Glasziou P, et al [webpage on the Internet]. The Oxford 2011 Levels of Evidence. Oxford Centre for Evidence-Based Medicine. Available from: http://www.cebm.net/index.aspx?o=5653. Accessed August 10, 2015. 
Journal of Pain Research

Dovepress

\section{Publish your work in this journal}

The Journal of Pain Research is an international, peer-reviewed, open access, online journal that welcomes laboratory and clinical findings in the fields of pain research and the prevention and management of pain. Original research, reviews, symposium reports, hypothesis formation and commentaries are all considered for publication.

The manuscript management system is completely online and includes a very quick and fair peer-review system, which is all easy to use. Visit http://www.dovepress.com/testimonials.php to read real quotes from published authors.

Submit your manuscript here: http://www.dovepress.com/journal-of-pain-research-journal 